\title{
A NADH Dehydrogenase Ubiquinone Flavoprotein is Decreased in Patients with Dilated Cardiomyopathy
}

\author{
Hitomi Ono ${ }^{1}$, Hiroshi Nakamura ${ }^{2}$ and Masunori Matsuzaki ${ }^{2}$
}

\begin{abstract}
Background It is known that patients with myocarditis of unknown etiology and with dilated cardiomyopathy show a high incidence of serum autoantibodies (M7) directed against mitochondrial flavoproteins. The tissue concentration of mitochondrial flavoproteins in the myocardium obtained from patients with dilated cardiomyopathy (DCM) was examined to further investigate the immunopathological mechanism of cardiomyopathy.

Methods and Results Myocardial tissue specimens were obtained from patients who underwent cardiac catheterization and a subendomyocardial biopsy for the diagnosis of cardiomyopathy. All samples were analyzed by Western blotting. NADH dehydrogenase ubiquinone flavoprotein (NDUFV1) production in the myocardium decreased significantly with DCM, in comparison to fumarate hydratase and flavoprotein SDHA. There was a significant negative-correlation between the left ventricular end-diastolic dimension and NDUFV 1 production $\left(\mathrm{R}^{2}=0.291\right.$, $\mathrm{p}$ value $\left.<0.05\right)$.

Conclusion NDUFV1 may be involved in the pathogenesis of DCM. A defect of mitochondrial NDUFV1 may reduce complex I, which produces most of the superoxide, which is then scavenged by the mitochondrial enzyme Mn-superoxide dismutase to produce $\mathrm{H}_{2} \mathrm{O}_{2}$. Exploring the nature of the candidate protein found in the myocardium in this study will provide further insight into the immunological mechanism of DCM.
\end{abstract}

Key words: flavoprotein, dilated cardiomyopathy, mitochondria

(Inter Med 49: 2039-2042, 2010)

(DOI: 10.2169/internalmedicine.49.3710)

\section{Introduction}

The relationship between myocarditis and dilated cardiomyopathy (DCM) remains controversial. A causal link between viral myocarditis and DCM has become evident owing to the tremendous development in the molecular analyses of autopsy and endomyocardial biopsy specimens, new techniques of viral gene amplification, and modern immunology research (1). An autoimmune response plays a key role in the progression after viral myocarditis and $\operatorname{DCM}(2,3)$. This occurs in the context of a polyclonal stimulation of the immune system after the initial viral assault that may have already been cleared when the autoreactive B- and T-cell response occurred. Repetitive coxsackievirus $\mathrm{B} 3$ (CVB3) infection produces cardiac dilatation without inflammatory cell infiltration in the heart in mice with postmyocarditis. In addition, autoimmunity mediated by certain circulating antibodies, e.g. antibodies against the CVB3 genome or a CVB3-related protein may play a role in the pathogenic mechanism for this phenomenon $(4,5)$. Flavoproteins are involved in a wide array of biological processes, including, but by no means limited to, bioluminescence, removal of radicals contributing to oxidative stress, photosynthesis, DNA repair, and apoptosis. Recently, Stähle et al reported that anti-mitochondrial flavoprotein autoantibodies are present in the serum of patients with myocarditis and DCM (6). To investigate the mechanism in these patients, the tissue concentration of NADH dehydrogenase ubiquinone flavoprotein (NDUFV1) of the mitochondrial Complex I, and SDHA of Complex II were examined using subendomyocardial biopsy samples in patients with DCM.

${ }^{1}$ Department of Medicine, Yamaguchi University, Ube and ${ }^{2}$ Division of Cardiology, Department of Medicine and Clinical Science, Yamaguchi University Graduate School of Medicine, Ube

Received for publication March 25, 2010; Accepted for publication July 6, 2010

Correspondence to Dr. Hiroshi Nakamura, nahirosi@yamaguchi-u.ac.jp 
Table 1. Characteristic of the Patients Involved in This Study

\begin{tabular}{ccccccccc}
\hline No. Pt. & Sex & Age & LVEDD & LVESD & LVEF & BNP & CRP & CPK \\
\hline 1 & M & 80 & 47 & 30 & 64 & 367 & 0.1 & 121 \\
2 & M & 60 & 72 & 66 & 25 & 192 & 0.2 & 56 \\
3 & F & 67 & 51 & 27 & 78 & 290 & 0.13 & 61 \\
4 & F & 45 & 57 & 45 & 50 & 183 & 0.02 & 166 \\
5 & F & 62 & 50 & 38 & 40 & 42 & 0.03 & 92 \\
6 & F & 39 & 63 & 56 & 25 & 856 & 0.62 & 97 \\
7 & M & 26 & 72 & 63 & 26 & 1905 & 1.55 & 132 \\
8 & M & 46 & 55 & 41 & 45 & 40 & 0.09 & 228 \\
9 & F & 72 & 63 & 54 & 35 & 202 & 0.02 & 97 \\
10 & M & 60 & 54 & 45 & 40 & 38 & 0.07 & 369 \\
11 & M & 50 & 71 & 67 & 25 & 1592 & 1.4 & 77 \\
12 & M & 50 & 47 & 25 & 78 & 111 & 0.06 & 63 \\
13 & M & 62 & 47 & 25 & 78 & 1678 & 4 & 182 \\
14 & F & 50 & 61 & 38 & 65 & 120 & 0.05 & 54 \\
\hline
\end{tabular}

LVEDD, left ventricular end-diastolic dimension (mm); LVESD, left ventricular end-systolic dimension (mm); LVEF, left ventricular ejection fraction (\%); BNP, brain natriuretic peptide (pg/ml); CRP, C-reactive protein (mg/dl); CPK, creatine phosphokinase (IU/L)

\section{Patients and Methods}

A total of 15 subjects (DCM, 14; control, 1) were enrolled in this study. All studies conformed to the Ethics Committee for Clinical Research at Yamaguchi University School of Medicine (No. H18-51: Proteomic analysis using endomyocardial samples of patients with dilated cardiomyopathy). Informed consent was obtained from all patients before this study. Fourteen patients (male/female: 8/6, mean age; 55 years) were admitted to the hospital for further examination for DCM (Table 1). The patients presented dilated hearts with systolic dysfunction and unexplained heart failure of variable duration in the absence of coronary artery or valvular heart diseases as documented by cardiac catheterization, echocardiography, myocardial scintigraphy, and/or coronary angiography. Four patients were diagnosed with post-myocarditis cardiomyopathy due to cell inflammation in the myocardium. Other patients displayed symptoms consistent with DCM. A Western blot analysis was used to examine the tissue concentration of fumarate hydratase, NDUFV1, and SDHA. We used a normal heart tissue as a control. Protein lysates were resolved by SDS-PAGE and electrotransferred to membranes. The membranes were incubated with anti-fumarate hydratase (C-16) (1:500; Santa Cruz Biotechnology, CA, USA), anti-NDUFV1 polyclonal (1:1,000; Abnova, Taiwan), and anti-SDHA (1:100,000; Abcam,Tokyo, Japan) followed by anti-mouse IgG conjugated with horseradish peroxidase. The significance of continuous variables, such as left ventricular end-diastolic dimension (LVEDD), left ventricular end-systolic dimension (LVEDSD), left ventricular ejection fraction (LVEF), and plasma brain natriuretic peptide (BNP) levels between the groups was analyzed using a two-way analysis of variance for repeated measures and Newmann-Keuls' post-hoc test. A $\mathrm{p}$ value of less than 0.05 was considered to be statistically significant.

Primary cultures of 1- to 2-day-old neonatal mouse ventricular myocytes were purchased form Primary Cell Co., Ltd. (Sapporo, Japan). Myocytes that were enzymatically dissociated were seeded on culture trays at a concentration of $3 \times 10^{5}$ cells $/ \mathrm{cm}^{2}$, and were incubated in Leibovitz L-15 Medium (Worthington Biochemical Co., Lakewood, NJ, USA) supplemented with $5 \%$ fetal bovine serum at $37^{\circ} \mathrm{C}$. Cultured myocytes were transferred on day 5 to serum-free medium, and exposed to the anti-NDUFV1 antibody for 24 hours. Primary antibody-bound NDUFV1 was visualized by oat anti-mouse IgG, DyLight 549 (USA), and examined under a confocal microscope (LSM 5, PASCAL). Samples processed without the primary antibody served as negative controls. The proportion of the NDUFV1 immunoreactive signal was defined as the number of high-signal-intensity (over $90 \%$ of the maximum level) pixels divided by the total number of pixels occupied by the myocytes.

\section{Results}

The production of myocardial NDUFV1 was significantly lower in patients with DCM, in comparison with the production of fumarate hydratase and flavoprotein SDHA $(\mathrm{p}<0.05)$ (Fig. 1A, B). There was also a negative correlation between the myocardial NDUFV1 level and LVEDD $\left(\mathrm{R}^{2}=0.291, \mathrm{p}<\right.$ 0.05 ) (Fig. 2A). There was no correlation between the myocardial NDUFV1 level and other markers, such as LVESD, LVEF, or plasma BNP, C reactive protein, and creatine phosphokinase level. In addition, no correlation was observed between the myocardial fumarate hydratase and flavoprotein SDHA level and other markers (Fig. 2B, C). In the cultured cardiomyocytes, anti-NDUFV1 antibody was located on the cell's cytoplasm but not on the nuclei 


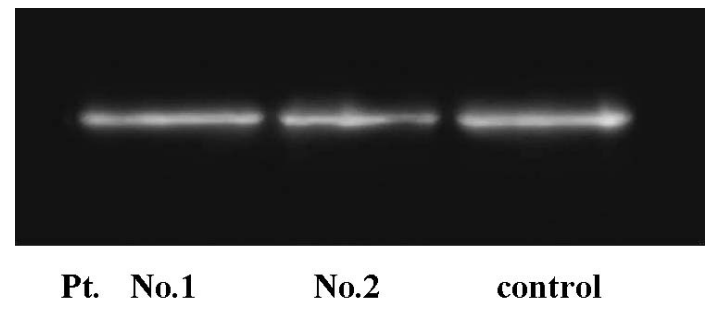

Figure 1A. Representative photograph of Western blotting used for NDUFV1.

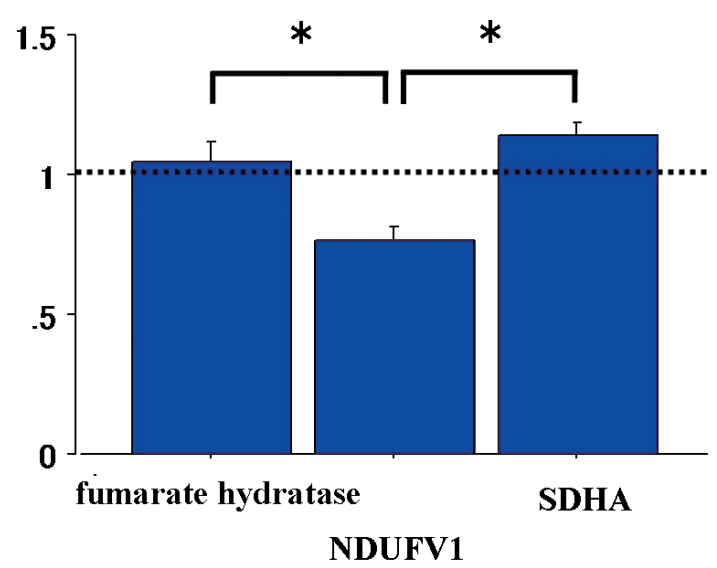

Figure 1B. A semiquantitative Western blot analysis of fumarate hydratase, NDUFV1, and flavoprotein SDHA in all patients. Production of myocardial NDUFV1 was significantly lower in this group compared to fumarate hydratase and flavoprotein SDHA. The dotted line indicates the control level. $\mathbf{p}<0.01$.

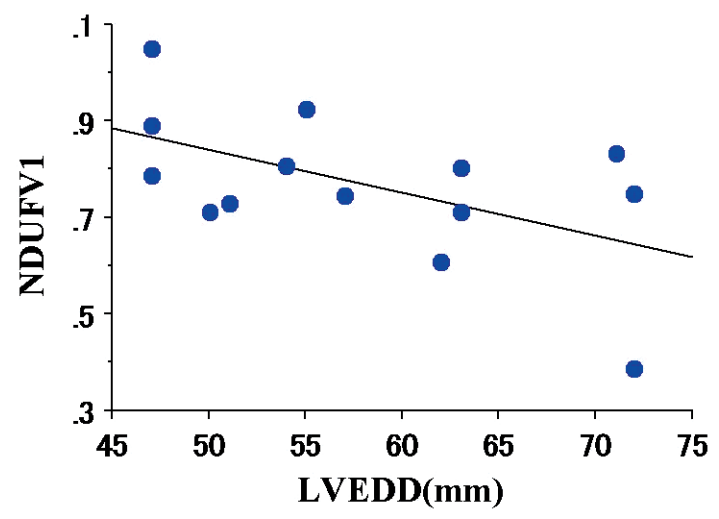

Figure 2A. Relationship between the myocardial NDUFV1 level and LVEDD ( $\mathrm{mm}$ ) in fourteen patients. There was a negative correlation between the myocardial NDUFV1 level and LVEDD. $R^{2}=0.291, p<0.05$.

(Fig. 2D).

\section{Discussion}

There is some clinical evidence that DCM is a late sequel of acute or chronic viral myocarditis. Infectious and autoim-

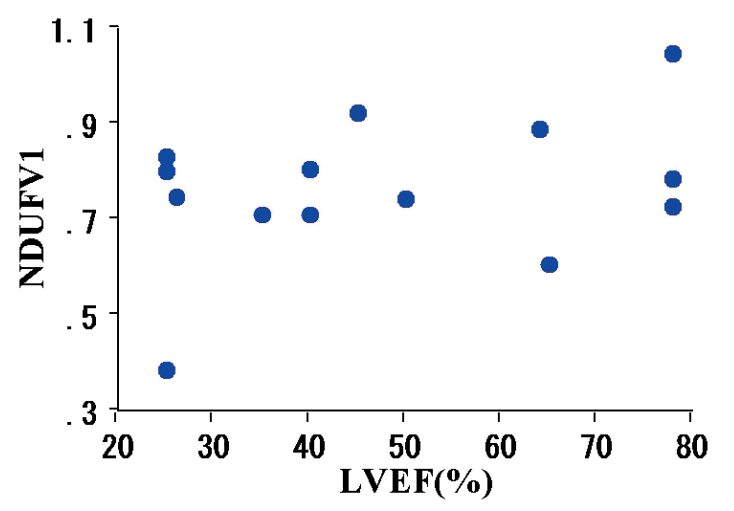

Figure 2B. Relationship between the myocardial NDUFV1 level and LVEF ( $\%$ ) in fourteen patients. There was no correlation between the myocardial NDUFV1 level and LVEF. $R^{2}=0.118, p<0.05$.

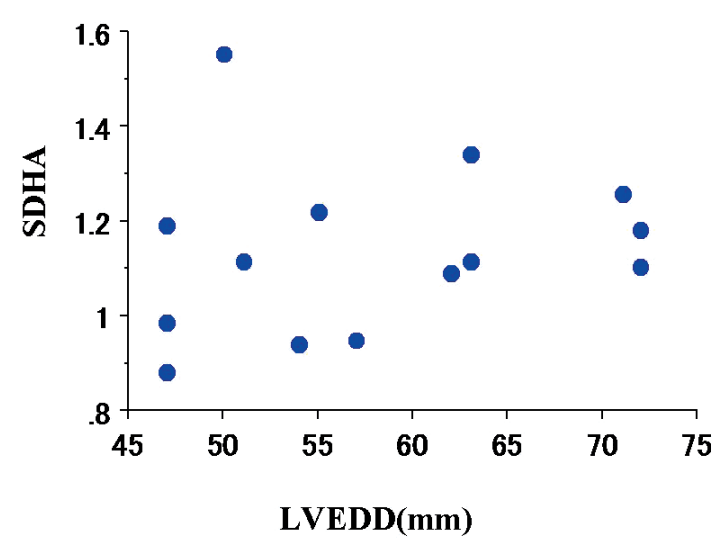

Figure 2C. Relationship between the myocardial SDHA level and LVEDD $(\mathrm{mm})$ in fourteen patients. There was no correlation between the myocardial SDHA level and LVEDD. $\mathbf{R}^{2}=\mathbf{0 . 0 3 2}, \mathrm{p}=\mathrm{NS}$.

mune myocarditis have also been extensively proven using murine and rat models. We previously demonstrated that repetitive CVB3 infection in mice could cause LV dilatation with dysfunction through autoantibodies, which were immunologically maximally activated at 2 weeks after the second CVB3 inoculation $(4,5)$. It is also known that patients with myocarditis of unknown etiology and with dilated cardiomyopathy show a high incidence of serum autoantibodies (M7) directed against mitochondrial flavoproteins (6). These results suggested that myocardial flavoproteins may therefore contribute to left ventricular dilatation in patients with DCM. Defects in the mitochondrial genome induce a heterogeneous array of clinical disorders, including mitochondrial cardiomyopathy. This suggests that increased production of reactive oxygen species (ROS) may be involved in mitochondrial cardiomyopathy, because mounting evidence has implicated ROS signaling in cardiac maladaptive remodeling (7). In the current study, none of the patients had a familial history or a phenotype associated with mitochondrial disorders. Moreover, the suppression of Complex I using an 


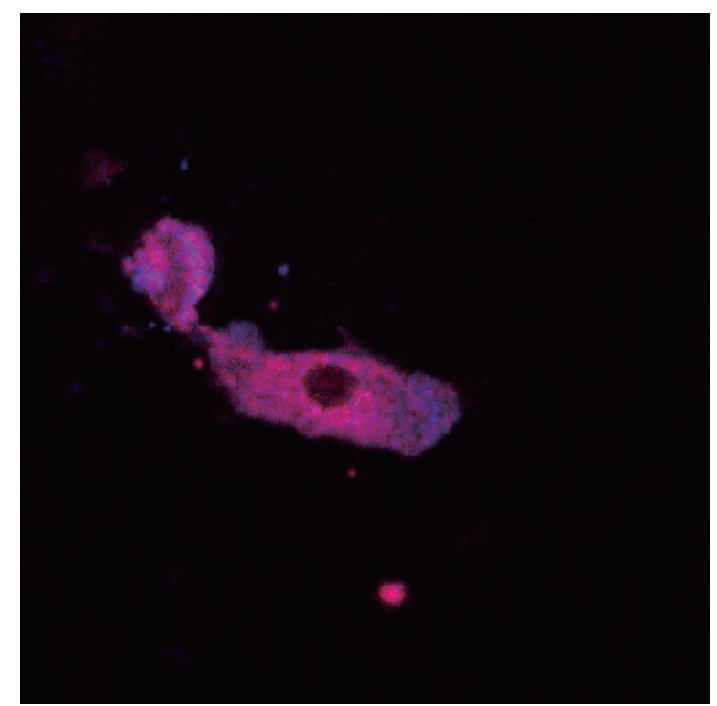

Figure 2D. Representative immunofluorescence images of cultured myocyte which were incubated with anti-NDUFV1 antibody are shown. Confocal images of NDUFV1 immunolabeling in cultured cardiomyocytes, which are present in the cell's cytoplasm but not in the nuclei.

anti-NDUFV1 antibody causes LV remodeling in normal mice. Complex I is also a significant source of ROS in the mitochondria, and its dysfunction has been implicated in a number of neuromuscular diseases such as Leigh syndrome and Parkinson's disease (8). Antibodies to mitochondrial proteins including the M7 protein have been found in patients with myocarditis and DCM (9). These antimitochondrial antibodies may bind flavoproteins and their precursors, such as Complex I, and inhibit their function. There is some evidence that patients have antibodies directed against a cytoplasmic or microsomal antigen, also present on the apical surface of the follicular epithelial cells, and it is now known to be thyroid peroxidase, the enzyme which iodinates thyroglobulin. Some residual thyroglobulin in the colloid and the acinar epithelial cells of the follicles has been stained by antibodies from a patient with Hashimoto's disease, which react with the cell's cytoplasm but not the nuclei (10). Therefore, our data are considered to be compatible with these results. We also investigated the mice with administration of anti-NDUFV1 antibody, and LVEDD were significantly higher, and the percent-fractional shortening was significantly lower than that of other groups (data not shown). Because of the small volume of biopsy sample, and few patients with DCM, we could not examine the serum titer of anti-NDUFV1 antibody nor mRNA in the myocardium, this is the first time to show that myocardial NDUFV1 was decreased using biopsy samples with DCM. Exploring the nature of the candidate proteins found in the myocardium, such as NDUFV1, will provide further immunological insight into the immunological pathogenesis of DCM.

\section{Acknowledgement}

This study was supported by grants-in-aid for research on intractable diseases, in Health and Labour Sciences Research Grants from Ministry of Health, Labour and Welfare, Japan.

\section{References}

1. Nakamura H, Yamamura T, Fukuta S, Matsumori A, Matsuzaki M. A pathogenic mechanism of chronic ongoing myocarditis. Jpn Circ J 60: 609-617, 1996.

2. Nakamura H, Yamaura $T$, Umemoto $S$, et al. Autoimmune response in chronic ongoing myocarditis demonstrated by heterotopic cardiac transplantation in mice. Circulation 94: 3348-3354, 1996.

3. Nakamura $H$, Kato $T$, Yamamura $T$, et al. Characterization of $T$ cell receptor $\beta$ chains of accumulating $\mathrm{T}$ cells in chronic onging myocarditis demonstrated by heterotopic cardiac transplantation in mice. Jpn Circ J 65: 106-110, 2001.

4. Nakamura H, Yamamoto T, Yamamura T, et al. Repetitive coxsackievirus infection induces cardiac dilation in post-myocarditic mice. Jpn Circ J 63: 794-802, 1999.

5. Takata $S$, Nakamura $H$, Umemoto $S$, et al. Identification of autoantobodies with the corresponding antigens for repetitive coxsackievirus infection-induced cardiomyopathy. Circ J 68: 677-682, 2004.

6. Stähle I, Brizzio C, Barile M, Bransch R. Anti-mitochondrial flavoprotein autoantibodies of patients with myocarditis and dilated cardiomyopathy (anti-M7): interaction woth flavin-carring proteins effect of vitamin B2 and epitope mapping. Clin Exp Immunol 115: 404-408, 1999.

7. Sebastiani M, Giordano C, Nediani C, et al. Induction of mitochondrial biogenesis is a maladaptive mechanism in mitochondrial cardiomyopathies. J Am Coll Cardiol 50: 1362-1369, 2007.

8. Barker C, Reda T, Hirst J. The flavoprotein subcomplex of complex I (NADH: ubiquinone oxidoreductase) from bovine heart mitochondoria: Insights into the mechanisms of NADH oxidation and $\mathrm{NAD}^{+}$reduction form protein film voltammetry. Biochemistry 46: 3454-3464, 2007.

9. Klein R, Maish B, Kochsiek K, Berg A. Demonstration of organ specific antibodies against heart mitochondoria (anti-M7) in sera from patients with some forms of heart disease. Clin Exp Immunol 58: 283-292, 1984.

10. Wraith DC. Immunological tolerance. In: Immunology. 6th ed. Roitt I, Brostoff J, Male D, Eds. Mosby, London, 2001: 401-402.

(C) 2010 The Japanese Society of Internal Medicine

http://www.naika.or.jp/imindex.html 